\title{
Contact Model And Simulation Analysis of Rail Grinding With Open-Structured Abrasive Belt Based On Pressing Plate
}

\section{Chang Qian}

Beijing Jiaotong University

\section{Zhiwei Wu}

Beijing Jiaotong University

Guangyou Hou

China Academy of Railway Sciences

Wengang Fan ( $\nabla$ wgfan@bjtu.edu.cn )

Beijing Jiaotong University https://orcid.org/0000-0002-3583-9936

Original Article

Keywords: Abrasive belt, Rail grinding, Pressing plate, Contact stress

Posted Date: August 6th, 2021

DOI: https://doi.org/10.21203/rs.3.rs-754322/v1

License: (c) (i) This work is licensed under a Creative Commons Attribution 4.0 International License. Read Full License 


\section{Title page}

\section{Contact Model and Simulation Analysis of Rail Grinding with Open-Structured Abrasive Belt Based on Pressing Plate}

Chang Qian, born in 1997, is currently a master candidate at Key Laboratory of Vehicle Advanced Manufacturing, Measuring and Control Technology, Ministry of Education, Beijing Jiaotong University, China.

Tel: 18810394615 ; E-mail: 19121248@bjtu.edu.cn

Zhi-Wei Wu, born in 1994, is currently a PhD candidate at Key Laboratory of Vehicle Advanced Manufacturing, Measuring and Control Technology, Ministry of Education, Beijing Jiaotong University, China.

E-mail: 1756832926@qq.com

Guang-You Hou, born in 1994, is currently an engineer at China Academy of Railway Sciences. He received his master degree on Mechanical Manufacturing and Automation in Beijing Jiaotong University, China, in 2020.

E-mail: 17121243@bjtu.edu.cn

Wen-gang Fan, born in 1985, is currently an associate professor at Beijing Jiaotong University. He received his PhD degree from Beijing Jiaotong University, China, in 2020. His main research direction is rail grinding technology and equipment, digital manufacturing technology and equipment.

E-mail:wgfan@bjtu.edu.cn

Corresponding author: Wen-gang Fan E-mail: wgfan@bjtu.edu.cn 


\title{
ORIGINAL ARTICLE
}

\section{Contact Model and Simulation Analysis of Rail Grinding with Open-Structured Abrasive Belt Based on Pressing Plate}

\author{
Chang Qian ${ }^{1,2} \cdot$ Zhiwei $\mathrm{Wu}^{1,2} \cdot$ Guangyou Hou ${ }^{3} \cdot$ Wengang Fan ${ }^{1,2}$
}

Received June xx, 201x; revised February xx, 201x; accepted March xx, 201x

(C) Chinese Mechanical Engineering Society and Springer-Verlag Berlin Heidelberg 2017

\begin{abstract}
Recently, the emerging rail grinding method with open-structured abrasive belt based on pressing plate which adopts full contour copying grinding method to extend the working length of abrasive belt and is more suitable for preventative grinding and maintenance of rail corrugation in high-speed railway has been proposed. The essence of its grinding process is the complex nonlinear interaction among the pressing plate, abrasive belt and rail. The research on the contact mechanism of the rail grinding method with open-structured abrasive belt based on pressing plate is still very lacking, which limits the practical application of the emering rail grinding method to a certain extent. In this paper, a theoretical model of the contact between pressing plate, abrasive belt and rail is established. The contact area morphology and contact stress distribution were obtained, and validity of the theoretical model was verified by finite element simulation. At the same time, the distribution characteristics of contact stress under single concentrated force, uniform force and multiple concentrated force were studied based on the contact model. It is concluded that the multiple concentrated force is the optimal loading method, and the contact stress is more uniform, which is more conducive to repair the standard contour of rail.
\end{abstract}

Keywords: Abrasive belt - Rail grinding - Pressing plate • Contact stress

Wen-Gang Fan

wgfan@bjtu.edu.cn

1 School of Mechanical, Electronic and Control Engineering, Beijing Jiaotong University, Beijing 100044, China

2 Key Laboratory of Vehicle Advanced Manufacturing, Measuring and Control Technology, Ministry of Education, Beijing 100044, China

3 Railway Engineering Research Institute,China Academy of Railway Sciences, Beijing 100081

\section{Introduction}

Rail grinding is currently recognized as a common means for daily maintenance of rail of various rail transit modes (high-speed rail, heavy load, subway, tram, etc.). By removing rail surface damage and defects and correcting rail contour, it can effectively improve wheel-rail matching relationship, restrain rolling contact fatigue, extend rail service life, reduce train running noise, improve train running stability, safety and passenger comfort, and bring significant social and economic benefits [1-3]. As a new method of rail grinding, rail grinding method with abrasive belt has attracted extensive attention in the world due to its advantages such as high grinding efficiency, flexible contact is not easy to damage rail, dust recovery is easy, safety and environmental protection, etc. [4].

Rail grinding with abrasive belt mainly include rail grinding with open-structured abrasive belt and rail grinding with closed-structured abrasive belt [5]. The wheel system for the open-structured abrasive belt includes the winding wheel, the releasing wheel, the idle wheel, and the tensioning wheel, as shown in Figure 1. The motor drives the winding wheel to rotate through a reduction mechanism, so that abrasive belt at the bottom of the pressing plate is slowly updated. At the same time, the whole device is moving at a uniform speed to ensure uniform grinding. It can greatly extend the working length of abrasive belt, significantly improve the grinding mileage, avoid frequent replacement of abrasive belt, and greatly improve the grinding quality and efficiency. 


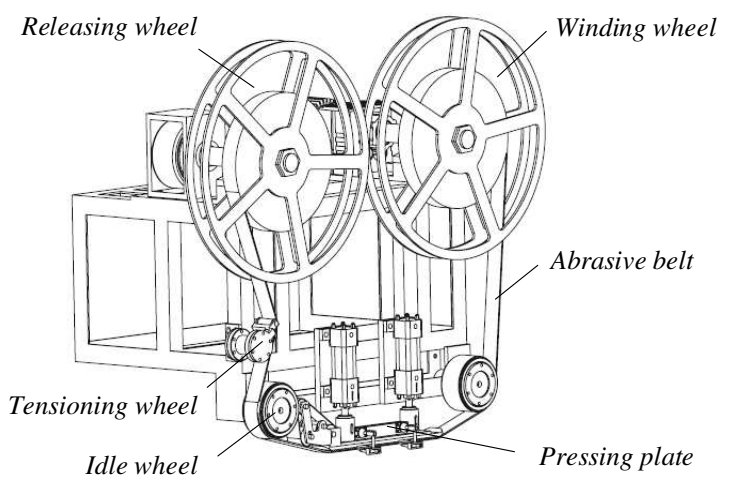

Figure 1 The principle of rail grinding with open-structured abrasive belt based on pressing plate

The process of rail grinding with open-structured abrasive belt based on pressing plate is essentially the complex nonlinear interaction among the pressing plate, abrasive belt and rail, which directly determines the grinding efficiency and grinding quality. The study of the contact relationship is the theoretical basis for the study of material removal mechanism, material temperature and material surface quality. Blum et al. [6] used the force balance equation and the minimum energy method to conduct research and regarded the contact between the contact wheel and the workpiece as Signorini contact, thus established an analytical model for quantitative grinding of abrasive belt. Ren et al. [7-8] and Zhang et al. [9-10] regarded the contact relationship between the contact wheel and the workpiece as Signorini contact problem to study the grinding operation process of grinding robot with abrasive belt. Khellouki et al. [11-12] studied the contact length between contact wheel with different hardness and cylindrical workpiece in rail grinding with open-structured abrasive belt, and established a contact model for qualitative analysis. Deresiewicz et al. [13] simplified the contact relationship between a flat contact wheel and the workpiece to be processed and regarded it as a simple contact behavior between a cylinder and a plane to establish a contact wheel friction model. Wang et al. [14] simplified the contact relationship of rail grinding with the closed-structured to the contact problem between two elastic cylinders, and then studied the contact behavior of the rail grinding with abrasive belt based on the elliptic Hertz contact theory. Wu et al. [15-19] also successively constructed a material removal model for precision grinding of flat contact wheel belt based on Hertz contact theory. Based on Hertz contact theory, Wang et al. [20-22] successively established the prediction model of abrasive belt wear, grinding power and material removal rate for rail grinding with abrasive belt based on flat contact wheel. Cheng et al. [23-24] established the contact model of rail grinding with closed-structured abrasive belt under static condition by applying elastoplastic theory and Hertz contact theory. The existing research mainly focus on rail grinding with closed-structured abrasive belt and rail grinding with open-structured abrasive belt based on contact wheel. Compared with rail grinding with closed-structured abrasive belt and rail grinding with open-structured abrasive belt based on contact wheel, the rail grinding with open-structured abrasive belt based on pressing plate adopts full contour copying grinding method to avoid over-grinding phenomenon in contact wheel abrasive belt grinding technology and extend the working length of abrasive belt, which is better applied in preventive grinding and maintenance of rail corrugation in high-speed railway. However, there is still a lack of research on the contact mechanism of rail grinding with open-structured abrasive belt based on pressing plate, which limits the practical application of the emerging rail grinding method to a certain extent.

Therefore, this paper will analyze the contact mechanism among the pressing plate, abrasive belt and rail, and establish the contact model, and explore the distribution law of the contact stress on the rail surface under different load modes. This will help to promote its engineering application and future development.

\section{Contact Model of Rail Grinding with Open-Structured Abrasive Belt based on Pressing Plate}

The research object of this paper is the macroscopic static contact behavior between the pressing plate and the rail contour. The microscale characteristics of the abrasive particles on the surface of abrasive belt and the influence of grinding process on the contact behavior is not taken into account. In the process of grinding, the pneumatic cylinder will press the pressing plate and abrasive belt on the surface of rail, the top surface of pressing plate is applied a concentrated force which is labeled F, the concentrated force can be transferred to the curved surface of rail through abrasive belt, so as to remove the material of rail and eliminate the surface disease of rail, as shown in Figure 2. 


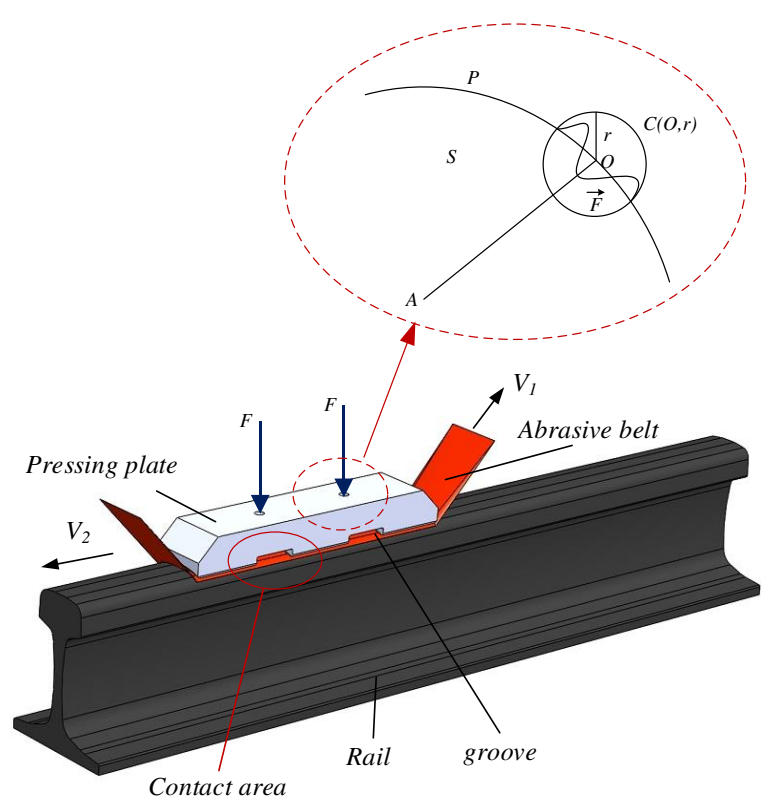

Figure 2 Schematic diagram of press pressing plate - abrasive belt - rail action

According to Saint-Venant's theory [25], $O$ is a point on the boundary $P$ of the elastomer $S$. Let $C(O, r)$ be a sphere centered at point $O$ and of radius $r$. Acting on $P \cap C(O, r)$ with a force system $F$ whose static force is equal to zero. If the ratio of $r / O A$ tends to zero, the stress distribution caused by $F$ tends to zero. When the thickness of pressing plate is limited and just around the position of the load (near the pressure position of pneumatic cylinder), the stress distribution of pressing plate can not be assumed to be the same everywhere. It is assumed that the concentrated force directly acts on the abrasive belt due to the limited thickness of the pressing plate, that is, the concentrated force generates point contact on abrasive belt. Rail arc surface is composed of arc segments with different radii. Let $I=1,2,3,4,5$ correspond to the left first arc (R13), the left second arc (R80), the middle arc (R300), the right second arc (R80) and the right first arc (R13), respectively. If the concentrated force $F$ of the air pressure bar is denoted as $F_{l}$ in the left arc in Figure 3, and when the concentrated force $F_{l}$ acts on the arc, the normal deviation Angle of the line between the point of application and the center of the arc is $\theta_{1}$, it can be known that the normal force acting on the left arc at this time is $F N_{l}=F_{l} \cos \theta$.

Hertz contact theory shows that the concentrated force applied to abrasive belt can form spherical deformation of radius $R_{I}$. The sphere is in contact with the arc surface of rail, resulting in contact stress between sphere and surface, and a cylinder of radius $R_{I}$ will be produced when acting on abrasive belt area. The outer curve of the rail can be viewed as part of another cylinder of radius $R_{2}$. At this time, the abrasive belt contact with rail surface can be converted into two cylinders contact. The normal force between the cylinders is $F_{N} E_{1}, v_{1}, E_{2}$ and $v_{2}$ are the elastic modulus and Poisson's ratio of cylinder 1 and cylinder 2, respectively. $R^{*}$ and $E^{*}$ are the effective sphere radius and the effective elastic modulus respectively, as shown in Eqs. (1) and (2). Set the direction along the rail as $x$ direction and the direction perpendicular to the rail as $y$ direction.

$$
\begin{gathered}
\frac{1}{E^{*}}=\frac{1-v_{1}^{2}}{E_{1}}+\frac{1-v_{2}^{2}}{E_{2}} \\
\frac{1}{R^{*}}=\frac{1}{R_{1}}+\frac{1}{R_{2}}
\end{gathered}
$$

According to Hertz theory, when two cylinders are in cross contact, as shown in Figure 3, the contact area is an ellipse. Since the contact area is an ellipse, the radius of the major axis is considered to be $a$ and the radius of the minor axis is considered to be $b$. According to contact mechanics [26] and elastic mechanics [27], Eqs. (3) and (4) expresses the size of the ellipse is:

$$
\begin{gathered}
a=\alpha^{3} \sqrt{F_{N} \frac{2 R_{1} R_{2}}{R_{1}+R_{2}}\left(\frac{1-v_{1}^{2}}{E_{1}}+\frac{1-v_{2}^{2}}{E_{2}}\right)} \\
b=\beta a
\end{gathered}
$$

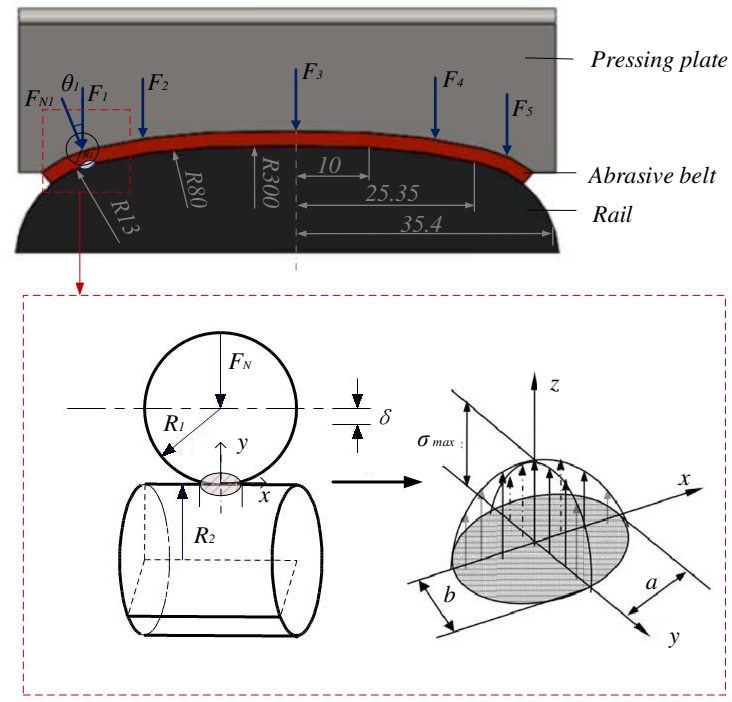

Figure 3 Cross contact of cylinder 
$P_{0}$ is the maximum crushing stress in the contact area.

Eq. (5) to calculate the maximum crushing stress.

$$
p_{0}=\frac{3 F_{N}}{2 \pi a b}
$$

The two cylinders have radius $R_{1}$ and $R_{2}$, the normal overlap is $\delta$ that are solved using Eq. (6). The pressure distribution in the contact area is shown in Eq. (7).

$$
\begin{gathered}
\delta=\lambda^{3} \sqrt{F_{N}^{2} \frac{2 R_{1} R_{2}}{R_{1}+R_{2}}\left(\frac{1-v_{1}^{2}}{E_{1}}+\frac{1-v_{2}^{2}}{E_{2}}\right)^{2}} \\
p\left(x_{i}, y_{i}\right)=p_{0} \sqrt{1-\frac{x_{i}^{2}}{a^{2}}-\frac{y_{i}^{2}}{b^{2}}}
\end{gathered}
$$

Wherein, the size coefficients of the elliptical contact area are $\alpha, \beta, \lambda$. It can be obtained by looking up Table 1 .

Table 1 Elliptic coefficient table

\begin{tabular}{cccccccc}
\hline $\boldsymbol{R}_{1} / \boldsymbol{R}_{2}$ & $\mathbf{1}$ & $\mathbf{3 / 2}$ & $\mathbf{2}$ & $\mathbf{3}$ & $\mathbf{4}$ & $\mathbf{6}$ & $\mathbf{1 0}$ \\
\hline$\alpha$ & 0.908 & 1.045 & 1.158 & 1.35 & 1.505 & 1.767 & 2.175 \\
$\beta$ & 1 & 0.765 & 0.652 & 0.482 & 0.400 & 0.308 & 0.221 \\
$\lambda$ & 2.080 & 2.060 & 2.025 & 1.950 & 1.875 & 1.770 & 1.613 \\
\hline
\end{tabular}

Then, under the action of normal force $F_{N l}$, the calculation method of deformation radius of abrasive belt $a 1$ is shown in Eq. (8).

$$
a 1=\sqrt[3]{F_{N 1} \frac{3 R^{*}}{4 E^{*}}}=\sqrt[3]{F_{1} \cos \theta_{1} \frac{3 R^{*}}{4 E^{*}}}
$$

According to Saint-Venant's principle, the deformation area of the abrasive belt is a cylindrical section of radius $a 1$, which crosses with the cylinder of the rail. According to the Hertz formula of cylindrical intersection, from Eqs. (1) to (7), the contact area and the contact stress of the abrasive belt and the rail in the left arc section can be respectively written as shown in Eqs. (9) to (13).

$$
\begin{gathered}
a_{11}=\alpha_{1} \sqrt[3]{F_{N 1} \frac{2 R_{1} R_{2}}{R_{1}+R_{2}}\left(\frac{1-v_{1}^{2}}{E_{1}}+\frac{1-v_{2}^{2}}{E_{2}}\right)} \\
b_{11}=\beta_{1} a_{11} \\
p_{01}=\frac{3 F_{N 1}}{2 \pi a_{11} b_{11}} \\
\delta_{1}=\lambda_{1} \sqrt[3]{F_{N 1}^{2} \frac{2 R_{1} R_{2}}{R_{1}+R_{2}}\left(\frac{1-v_{1}^{2}}{E_{1}}+\frac{1-v_{2}^{2}}{E_{2}}\right)^{2}} \\
p_{1}\left(x_{i}, y_{i}\right)=p_{01} \sqrt{1-\frac{x_{i}^{2}}{a_{11}^{2}}-\frac{y_{i}^{2}}{b_{11}^{2}}}
\end{gathered}
$$

Similarly, for the left second arc and the middle arc, the contact area and contact stress between the abrasive belt and the rail can be obtained respectively. Adopting tensor form, the contact theory of each arc can be summarized as shown in Eqs. (14) to (18).

$$
\begin{array}{r}
a_{I i}=\alpha \sqrt[3]{F_{N i} \frac{2 R_{1} R_{2}}{R_{1}+R_{2}}\left(\frac{1-v_{1}^{2}}{E_{1}}+\frac{1-v_{2}^{2}}{E_{2}}\right)} \\
b_{I i}=\beta_{I} a_{I i} \\
p_{o i}=\frac{3 F_{N i}}{2 \pi a_{I i} b_{I i}} \\
\delta_{i}=\lambda_{I} \sqrt[3]{F_{N i}^{2} \frac{2 R_{1} R_{2}}{R_{1}+R_{2}}\left(\frac{1-v_{1}^{2}}{E_{1}}+\frac{1-v_{2}^{2}}{E_{2}}\right)^{2}} \\
p_{i}\left(x_{i}, y_{i}\right)=p_{0 I} \sqrt{1-\frac{x_{I}^{2}}{a_{I i}^{2}}-\frac{y_{I}^{2}}{b_{I i}^{2}}}
\end{array}
$$

Where, $I=1,2,3,4,5$, corresponds to the left first arc, the left second arc, the middle arc, the right second arc and the right first arc respectively. So far, we have determined the contact model between the abrasive belt and the rail. 


\section{Simulation Verification of Contact Model of Rail Grinding with Open-Structured Abrasive Belt based on Pressing Plate}

The contact problem among pressing plate, abrasive belt and rail is a highly nonlinear problem, so this paper chooses ABAQUS software to analyze the contact problem. For China high-speed railways (CHSR), the wavelength of rail corrugation for high-speed running situation is usually between $120 \mathrm{~mm}$ and $150 \mathrm{~mm}$. In order to achieve effective removal of the corrugation on the basis of ensuring the flexibility of the grinding components in the bend, the length of pressing plate is designed as $340 \mathrm{~mm}$. The width of the embossed plate is set to $70 \mathrm{~mm}$ for maximum envelope range. According to Wang's research [28], in order to promote the heat dissipation of rail surface and avoid the abrasive debris adhering to the surface of abrasive belt which weakens material removal properties, at least two grooves are made on the bottom surface of pressing plate, and the width of the grooves is $5 \mathrm{~mm}$ to $30 \mathrm{~mm}$.

It is assumed that the width of groove is $30 \mathrm{~mm}$ and the number of groove is 2 , and this contour parameter is used in the subsequent contact stress simulation calculation. The $60 \mathrm{~kg} / \mathrm{m}$ rail was selected for the rail model. In order to improve the calculation efficiency, save the calculation cost and ensure the accuracy of the results, it is necessary to refine the mesh models of abrasive belt, the upper surface of rail and the lower surface of pressing plate. In order to avoid malformation or irregularity during meshing, which will affect the simulation accuracy, the thickness of grid cannot be changed suddenly, so the mesh transition strategy is adopted.

The $60 \mathrm{~kg} / \mathrm{m}$ rail material is high manganese steel, belt material is rubber. The rail is defined as elastoplastic material and the abrasive belt as elastic material. The material parameter settings of each model are shown in Table 2.

Compared with rail grinding based on contact wheel, the rail grinding with abrasive belt based on pressing plate adopts copying contact, and the contact area is far larger than rail grinding based on contact wheel. Combined with the actual working conditions of high-speed grinding, the load mode is selected as single concentrated force. The force is applied in the center of pressing plate and the direction is vertically downward.
Table 2 Material parameters

\begin{tabular}{|c|c|c|c|}
\hline Name & Rail & The abrasive belt & The pressing plate \\
\hline $\begin{array}{c}\text { Elastic } \\
\text { modulus (Mpa) }\end{array}$ & $2.1 \mathrm{e} 5$ & 7.8 & $2.1 \mathrm{e} 5$ \\
\hline $\begin{array}{l}\text { Density } \\
\left(\mathrm{kg} \cdot \mathrm{m}^{-3}\right)\end{array}$ & 7800 & 2000 & 7800 \\
\hline Poisson's ratio & 0.3 & 0.22 & 0.3 \\
\hline $\begin{array}{c}\text { Specific heat } \\
\text { capacity }(\mathrm{kg} \cdot \mathrm{K})\end{array}$ & 460 & 93 & 460 \\
\hline $\begin{array}{c}\text { Young's } \\
\text { modulus (Gpa) }\end{array}$ & 200 & 0.03 & 200 \\
\hline
\end{tabular}

Six grinding pressure values were selected, and the contact stress of the theoretical model was calculated by Mathematica software, as shown in Table 3.

Table 3 Theoretical calculation results of contact stress under different grinding pressures

\begin{tabular}{ccccccc}
\hline$F(\mathrm{~N})$ & 600 & 800 & 1000 & 1200 & 1400 & 1600 \\
\hline$P(\mathrm{Mpa})$ & 0.157 & 0.179 & 0.209 & 0.236 & 0.257 & 0.289 \\
\hline
\end{tabular}

The finite element simulation results corresponding to the theoretical model were obtained to compare the simulation results with the theoretical values. Contact stress nephogram under different grinding pressures is shown in Figure 4. The contact stress increases with the increase of grinding pressure, mainly because the increase of contact pressure makes the contact stress at the application point increase.
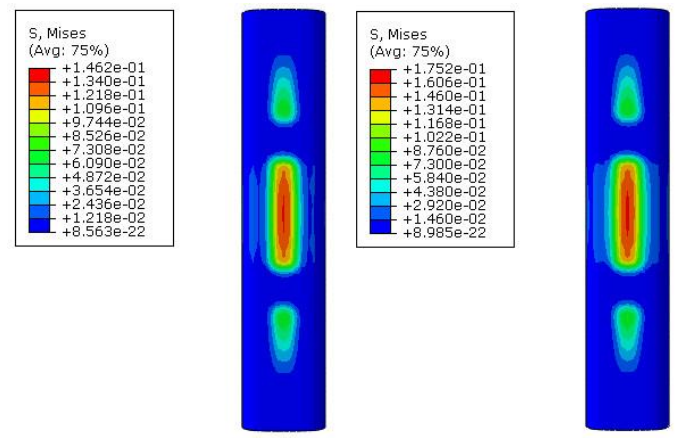
(a) $600 \mathrm{~N}$
(b) $800 \mathrm{~N}$ 

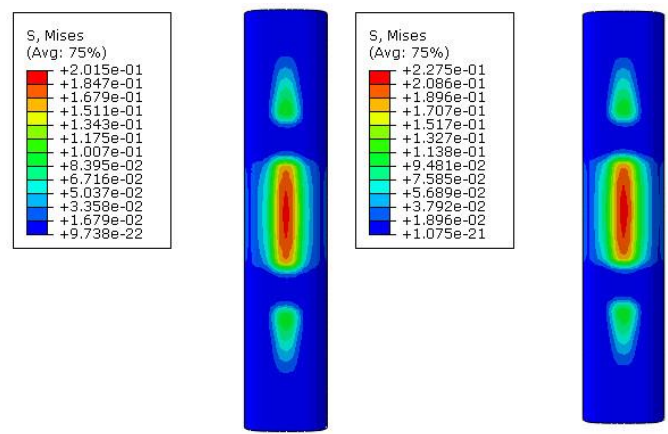

(c) $1000 \mathrm{~N}$
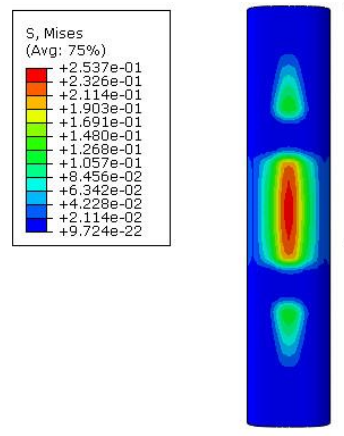

(d) $1200 \mathrm{~N}$

(e) $1400 \mathrm{~N}$

(f) $1600 \mathrm{~N}$

Figure 4 Contact stress nephogram of rail surface

In order to intuitively compare and analyze the contact stress matching between the finite element simulation model and the theoretical model under different pressures, the contact stress at the same position on the rail surface was extracted. The contact stress was compared respectively between the finite element simulation results and the theoretical calculation results, as shown in Figure 5. The contact stress is positively correlated with the grinding pressure and gradually increases with the increase of grinding pressure. According to analyze the data of the finite element simulation model and the simulation model, the maximum error is $7.3 \%$, which may be due to the error caused by the mesh division and calculation of the finite element simulation software. The simulation results are in good agreement with the theoretical calculation values. The correctness of the contact model of pressing plate-abrasive belt-rail under concentrated force pressure is verified.

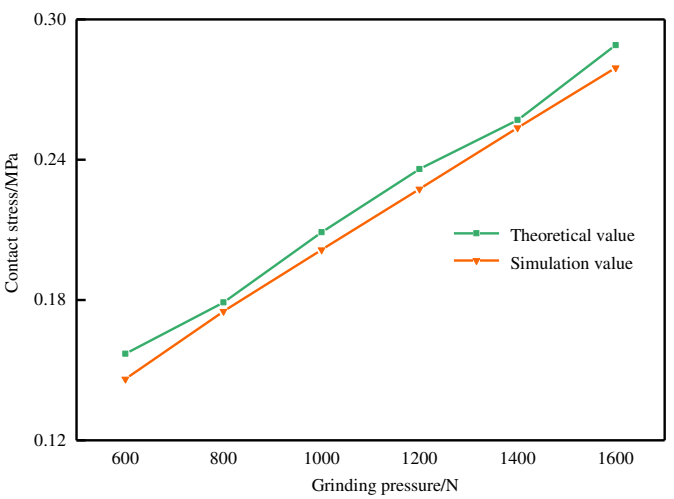

Figure 5 Comparison and analysis of contact stress between theoretical values and simulation values

\section{Comparative Analysis of the Influence of Different Load Modes on Contact Stress}

Since the thickness of pressing plate is limited, it is assumed that the concentrated force acts directly on the abrasive belt and forms point-surface contact according to Saint Venant theory and small deformation hypothesis. Under the action of external force, the internal stress of pressing plate is mainly concentrated in the force position, resulting in the phenomenon that the stress on the rail surface is too concentrated. By adjusting load mode, the contact stress distribution with the rail can be relatively uniform, and the problem of the contact stress concentration on the rail surface can be improved. Otherwise, the unreasonable load mode will lead to a larger contact stress at the rail surface position corresponding to the force position of pressing plate, resulting in more material removal at this position and a large difference between the rail profile after grinding and the standard profile, resulting in multiple point contact, noise, and even affecting the safety of the train operation. Therefore, it is very important to analysis of the influence of different load modes on contact stress.

In this cross-section, finite element simulation analysis will be carried out on the contact behavior under single concentrated force, uniform force and multiple concentrated force loads respectively, and the influence of different load modes on the contact stress of rail surface will be compared, which lays a foundation for the selection and optimization of load modes of rail grinding with open-structured abrasive belt based on pressing plate. 


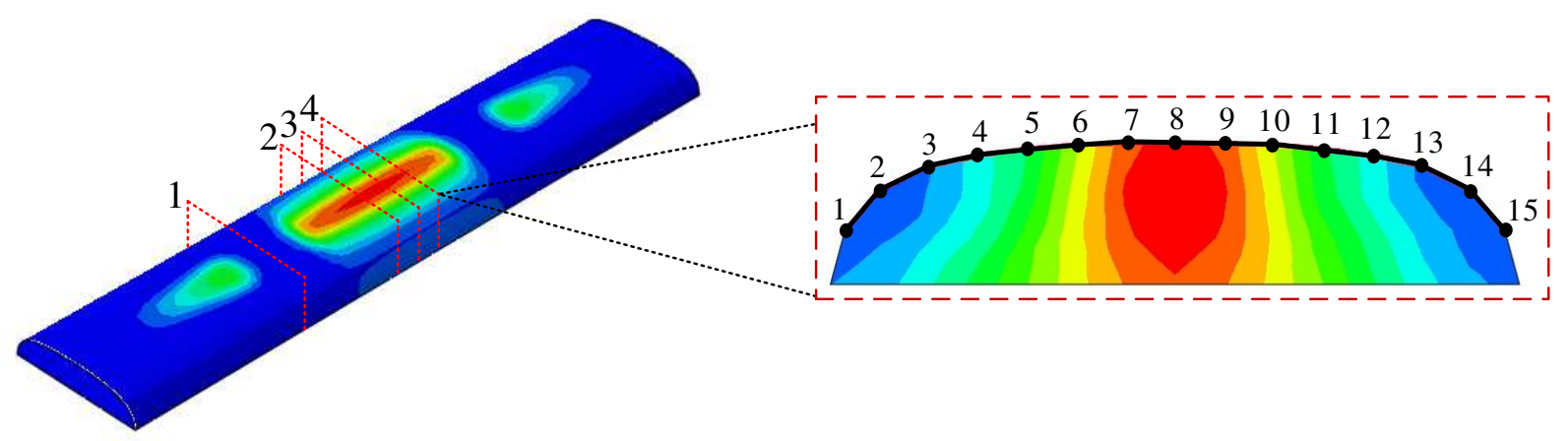

Figure 6 Schematic diagram of cross-section and node

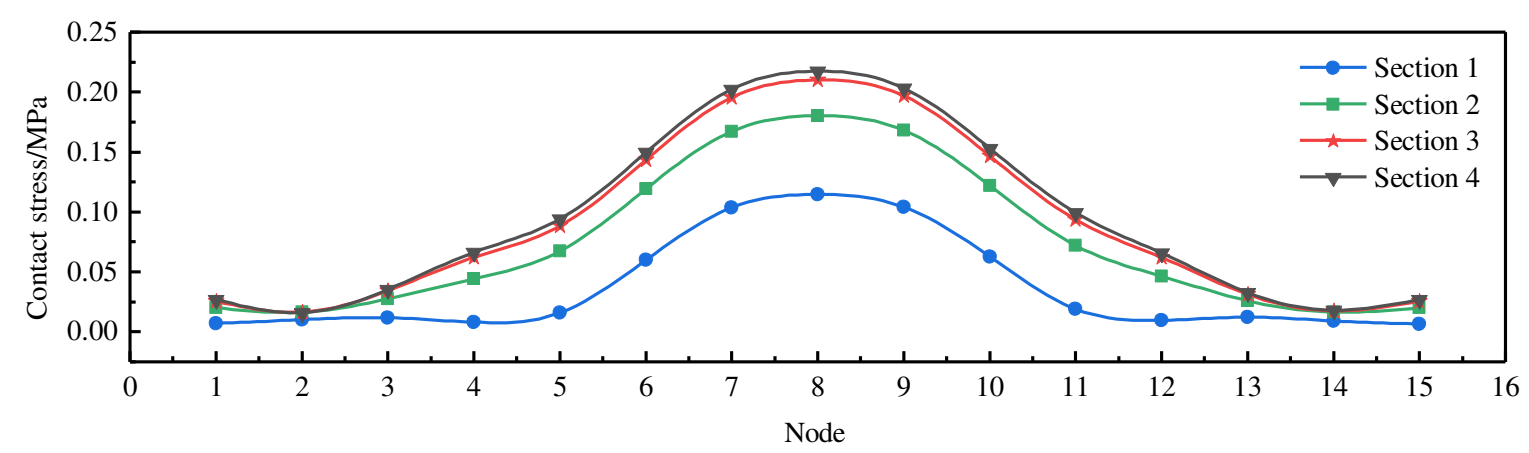

Figure 7 Contact stress nephogram of cross-section under single concentrated force

\subsection{Contact Stress Distribution under Single Concentrated Force}

Combined with the actual working conditions of high-speed grinding, the single concentrated force with grinding pressure of $1200 \mathrm{~N}$ is selected, and the force is applied in the center of pressing plate and the direction is downward. From the Figure 6, the contact stress on the rail surface is symmetric with respect to the middle cross-section 4. In order to directly analyze the stress distribution on the rail surface in the direction parallel to the cross-section under a single concentrated force, four cross-sections parallel to the rail were selected to extract the contact stress at 15 points on the cross-section, as shown in Figure 7.

Figure 7 shows that the contact stress is concentrated in the middle of rail, the contact stress presents an inverted triangle distribution along the contact area on both sides of the rail direction. The contact stress distribution at different sections is similar, and the magnitude is obviously different. The maximum contact stress appears at the 8 node of cross-section 4, the maximum contact stress is $0.217 \mathrm{MPa}$, and the average contact stress of cross-section is $0.094 \mathrm{MPa}$.

\subsection{Contact Stress Distribution under Uniform Force}

Combined with the actual working conditions of high-speed grinding, uniformly distributed load on the surface of pressing plate is set to $0.05 \mathrm{~N} / \mathrm{mm}^{2}$, and other simulation parameters of single concentrated force is the same.

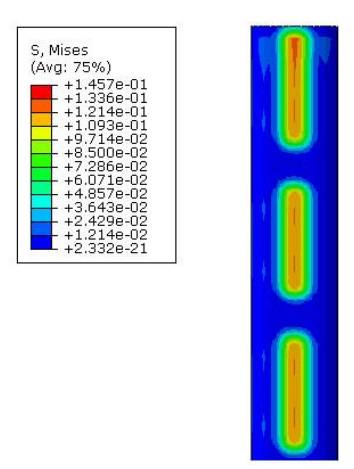

Figure 8 Contact stress nephogram of rail surface 


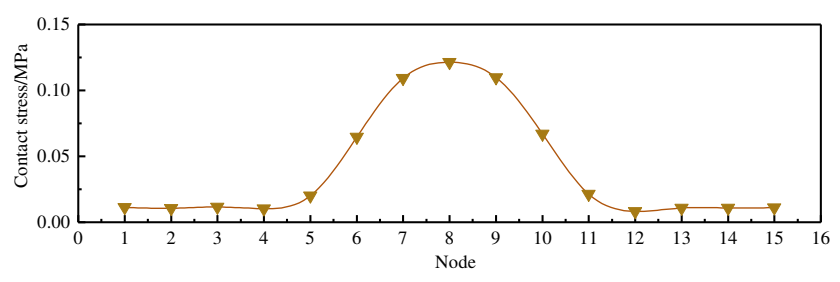

Figure 9 Contact stress nephogram of cross-section under uniform force

Figure 8 shows that the contact stress on the rail surface presents non-uniform distribution, and is divided into three areas. The shape of the contact area is similar, and all of them are long strips. There are two red areas of stress concentration on the symmetrical sides of the center of each contact stress distribution region, and the two ends are hemispherical. The force is mainly concentrated on the surface of the rail with the curvature of $300 \mathrm{~mm}$, and the contact stress of the rail contour with the curvature of $80 \mathrm{~mm}$ and $13 \mathrm{~mm}$ is very small, because the distance of transmission force is the shortest and the profile interference is the largest when the curvature of rail is $300 \mathrm{~mm}$ under the uniform force. Figure 9 shows that the contact stress on the cross-section presents symmetrical distribution and gradually decreases from the middle to the side. The contact stress on the middle node 8 is the maximum, and the maximum contact stress is $0.121 \mathrm{MPa}$, and the contact stress on the side is about $0.011 \mathrm{MPa}$.

\subsection{Contact Stress Distribution under Multiple Concentrated Force}

Based on single concentrated load mode, the contact stress distribution under multiple concentrated load mode is studied. The number of the groove on the bottom of pressing plate is 2 , and the working surface of the grinding rail is divided into three contact surfaces by the grooves. The point of force application along the direction of rail can be selected in the middle of each contact surface, which is conducive to more uniform distribution of contact stress. The diameter of the contact surface between pneumatic cylinder and pressing plate is $5 \mathrm{~mm}$, but the diameter of pneumatic cylinder is $40 \mathrm{~mm}$, and the point of force application to the cross-section is limited, so each contact surface to select two symmetrical force points. To sum up, the number of point of force application at all contact surfaces is selected as 6 in the multiple concentrated force. In order to compare the influence of the force position on the contact stress under multiple concentrated forces, the positions 1-7 of multiple concentrated forces are marked. The force position 1 is closest to the middle of pressing plate, and the force position 7 is closest to the edge of pressing plate, as shown in Figure 10. In the case of multiple concentrated force, each force is set to $200 \mathrm{~N}$, and other parameters are set the same as the simulation parameters of single concentrated force. The contact stress distribution nephogram under multiple concentrated force is shown in Figure 11. The method of data extraction is similar to single concentrated force and uniform force. The cross-section at the middle position of rail stress area was selected to extract the contact stress of 15 nodes on the cross-section, as shown in Figure 12.

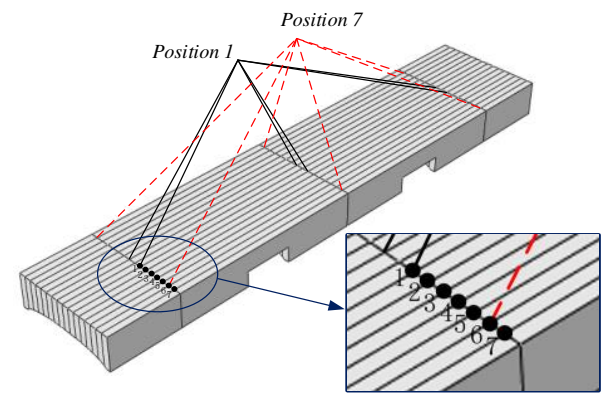

Figure 10 Position of multiple concentration force application
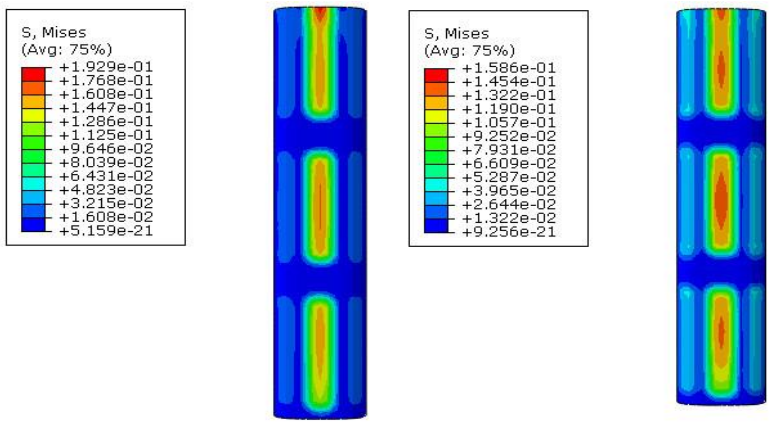

(a) Force position 1

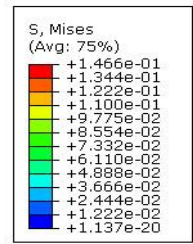

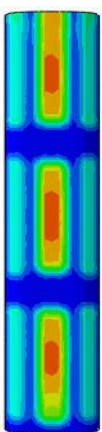

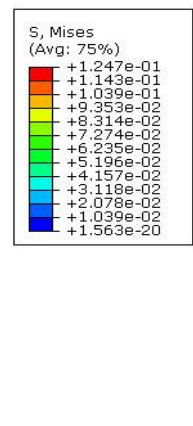

(b) Force position 2

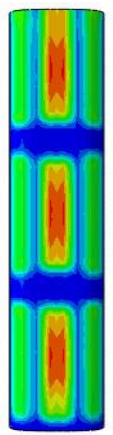

(c) Force position 3

(d) Force position 4 

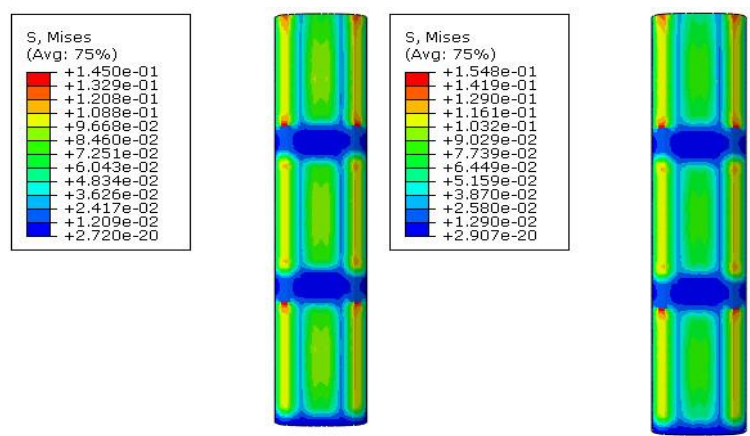

(e) Force position 5

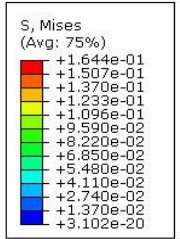

(g) Force position 7

Figure 11 Distribution of contact stress at different force position

According to the stress nephogram, the overall shape of the contact stress distribution is the same as that of the contact area of pressing plate, which is divided into three rectangular areas, and each rectangular area is divided into three strips. The junction of rail curvature of $300 \mathrm{~mm}$ and $80 \mathrm{~mm}$ is not as smooth as the curved surface after meshing, which leads to uneven forces on the two contact surfaces, and finally leads to uneven contact stress distribution and thin strip area with slightly lower contact stress. For the contact stress on the rail surface at positions 1 to 7 , the concentration area of contact stress changes correspondingly as the force position moves to both sides. Judging from the Figure 12, the contact stress on the cross-section is wavy as a whole, with three peaks and two troughs. The peaks of nodes 1-5 correspond to the rail curvature of $300 \mathrm{~mm}$ and the rail curvature of 80 $\mathrm{mm}$, and the troughs of nodes 5 and 11 correspond to the junction of rail curvature of $300 \mathrm{~mm}$ and $80 \mathrm{~mm}$. The maximum contact stress at the position $1 \sim 2$ appears at node 8 , while the maximum contact stress at the position 2 7 appears at node 7 or 10 and the stress in the center area is not the maximum. This is due to the fact that the two force points at the position 1 2 are close to each other and the stress centers overlap, while there is a certain distance between the two force points at the position 2 7. Combined with the influence of the rail contour, there is a phenomenon of stress reduction at the wave peak.

To evaluate the stress uniformity is to evaluate the stress fluctuation and dispersion of the joint of rail surface. The value of contact stress fluctuation is smaller, the contact stress of rail surface is more uniform. The statistical method were used to evaluate the contact stress on the rail surface, and the contact stress node data extracted from the cross-sections at each force application position were sorted out and the line chart was made. As can be seen from the Figure 13, when the force position moves from the middle to the side, both range and variance decrease first and then increase.

The range and variance of the force position 5 are the least, so position 5 is the best force position where the contact stress is relatively uniform under the multiple concentrated force.

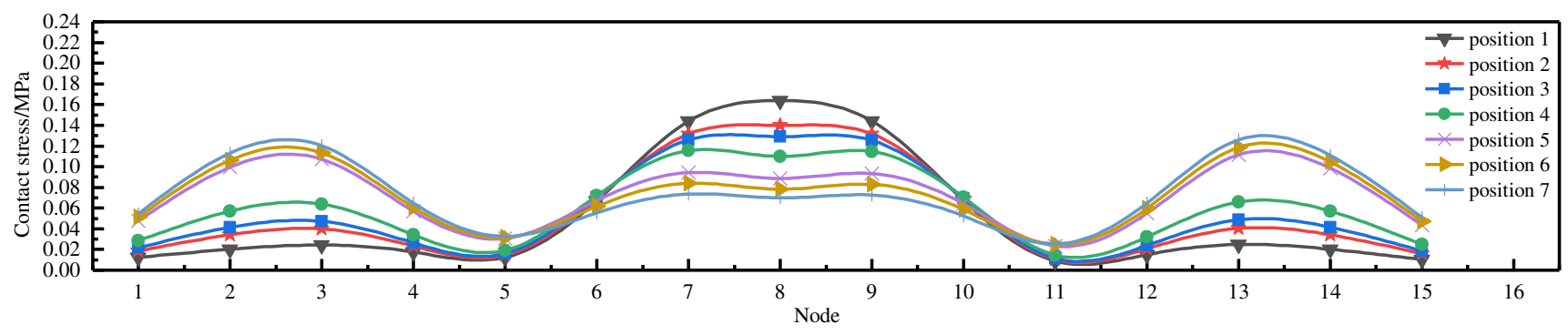

Figure 12 Simulation of different force position 


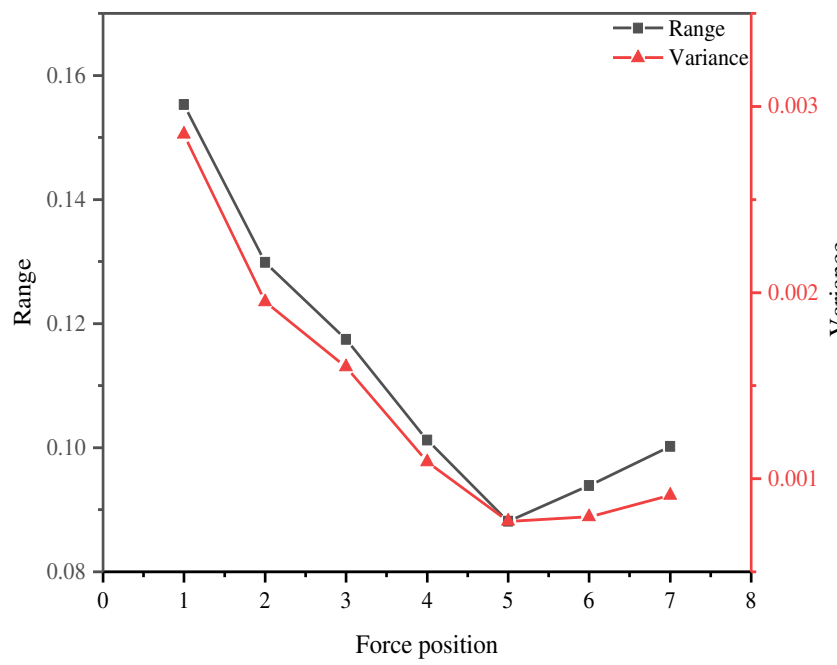

Figure 13 Range and variance of different force position

\subsection{Comparison of Contact Stress under Different Load Modes}

In order to ensure the comparability of data, the same cross-section is selected to ensure that the stress nodes on the cross-section are completely consistent when extracting the contact stress data of three load modes. Since a relatively optimal multiple concentrated force position is obtained, so the extracting data in the position 5 of multiple concentrated force.

The value of contact stress under three load modes was extracted respectively, and the data of the same cross-section and the same stress node were obtained to make the stress change curve, as shown in Figure 14.

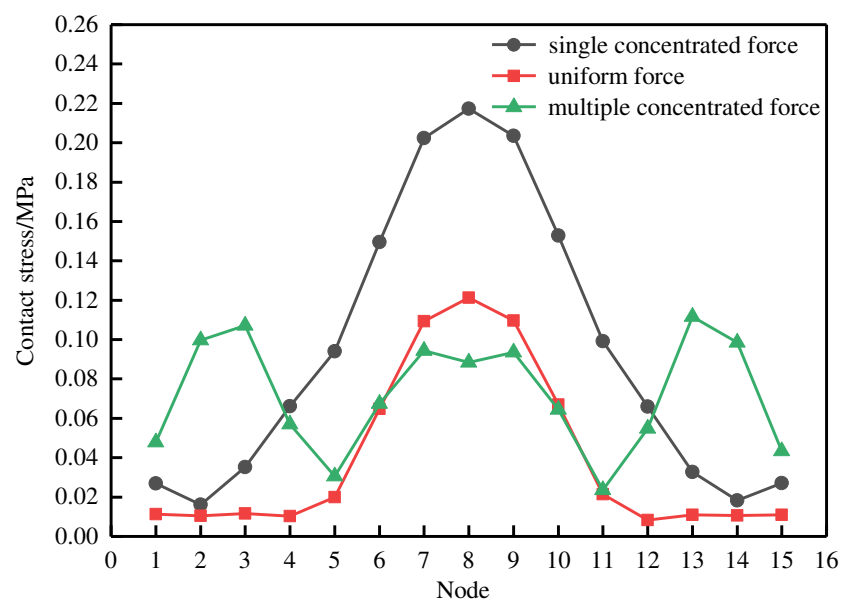

Figure 14 Stress curves of nodes with different application modes
Table 4 Cross-section stress analysis table under different loading modes

\begin{tabular}{cccc}
\hline & $\begin{array}{c}\text { Single } \\
\text { concentrated } \\
\text { force }\end{array}$ & $\begin{array}{c}\text { Uniform } \\
\text { force }\end{array}$ & $\begin{array}{c}\text { multiple } \\
\text { concentrated } \\
\text { force }\end{array}$ \\
\hline $\begin{array}{c}\text { average } \\
\text { standard deviation }\end{array}$ & 0.093787 & 0.039887 & 0.072176 \\
variance & 0.073232 & 0.042509 & 0.028739 \\
maximum & 0.005005 & 0.001687 & 0.000771 \\
minimum & 0.217368 & 0.121335 & 0.111701 \\
range & 0.016046 & 0.008296 & 0.023544 \\
\hline
\end{tabular}

Figure 14 shows that three kinds of load mode, rail showed different distribution of contact stress of the same cross-section. In order to compare the stress uniformity of the cross-section under three loading modes, the contact stress of the cross-section under different loading modes is analyzed (such as in Table 4) and make the range and variance change curve, as shown in Figure 15.

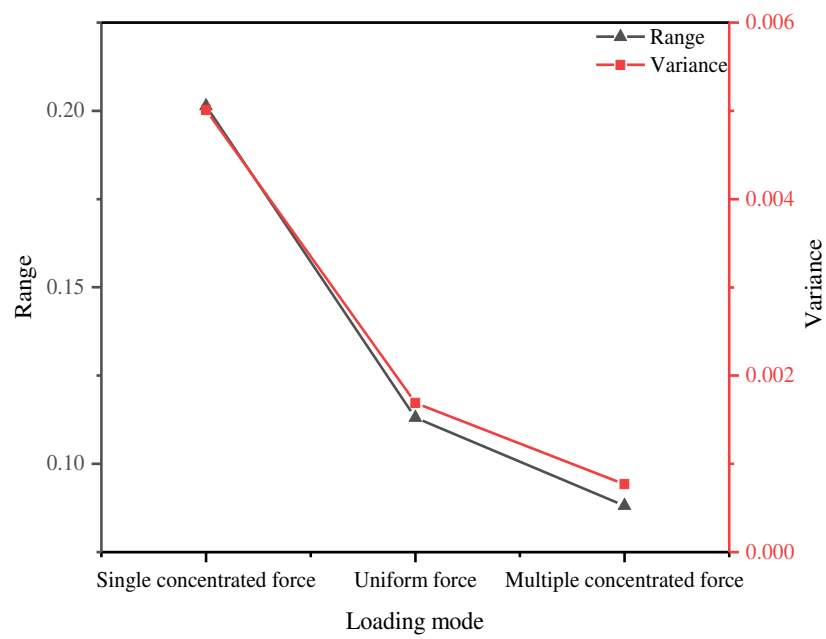

Figure 15 Range and variance of different loading modes

Figure 15 shows that compared with single concentrated force and uniformly force, the range and variance of the contact stress on the rail surface under the multiple concentrated force are the smallest. This indicates that the contact stress on the surface of the rail is more uniform under the load mode of multiple concentrated force, which is conducive to the repair of the standard contour of the rail and avoids the phenomenon that the contact stress concentration only removes the material from a single position of the rail.

\section{Conclusions}

In this paper, the contact behavior of rail grinding with open-structured abrasive belt based on pressing plate is 
studied. The conclusion can be drawn as:

(1) The contact relationship among the pressing plate, abrasive belt and rail is analyzed, and the contact model is established. The contact area morphology and contact stress distribution were obtained, and the validity of the theoretical model was verified by finite element simulation. The maximum error was $7.3 \%$.

(2) In the case of single concentrated force, the contact stress is concentrated in the middle of the rail, and the contact stress presents an inverted triangle distribution along the rail direction. The contact stress area is small and concentrated on the top of the rail surface under uniform force. The shape of the contact stress distribution under multiple concentrated force is the same as that of the contact area between the abrasive belt and the pressing plate, which is divided into three rectangular areas, and each rectangular area is divided into three strips.

(3) The contact stress values of 15 identical stress nodes on the same cross-section parallel to the rail are extracted. After range and variance analysis, it is known that multiple concentration force is the optimal load mode, and force position 5 is the optimal force position under multiple concentration force.

\section{Declaration}

\section{Acknowledgements}

Thanks for the support of computing resources provided by Key Laboratory of Vehicle Advanced Manufacturing, Measuring and Control Technology, Ministry of Education, Beijing Jiaotong University, China.

\section{Funding}

The authors would like to thank the financial support from the Fundamental Research Funds for the Central Universities (Grant No. 2019JBM050).

\section{Availability of data and materials}

The datasets supporting the conclusions of this article are included within the article.

\section{Authors' contributions}

The author' contributions are as follows: Chang Qian and Guang-You Hou was in charge of the whole modeling and simulation analysis; Chang Qian and Zhi-Wei Wu wrote the manuscript. Wen-Gang Fan checked the grammar of the paper and provided testing equipment for testing.

\section{Competing interests}

The authors declare no competing financial interests.

\section{Consent for publication}

Not applicable

\section{Ethics approval and consent to participate}

Not applicable

\section{References}

[1] S D Zhi, J Y Li, A M Zarembski. Grinding motor energy saving method based on material removal model in rail grinding processes. International Journal of Precision Engineering and Manufacturing-Green Technology, 2015, 2(1).

[2] W G Fan, Y M Liu, J Y Li. Development status and prospect of rail grinding technology for high speed railway. Journal of Mechanical Engineering, 2018, 54(22): 184-193.

[3] W G Fan, W X Wang, G Y Hou, et al. Macro Contact Pressure Modeling and Simulation for Rail Grinding with Abrasive Belt Based on Curvature Match. Journal of Mechanical Engineering, 2020, 56(02): 154-162.

[4] W G Fan, J F Cheng, Y F Wu, et al. Research on static contact behavior and simulation for rail grinding by abrasive belt based on concave type contact wheel. Journal of Mechanical Engineering, 2018, 54(14): 152-158.

[5] Y J Wang, Y Huang, Y X Chen, et al. Model of an abrasive belt grinding surface removal contour and its application. The International Journal of Advanced Manufacturing Technology, 2016, 82(9-12).

[6] $\mathrm{H}$ Blum, F $\mathrm{T}$ Suttmeier. An adaptive finite element discretisation $\uparrow$ for a simplified Signorini problem. Calcolo, 2000, $37(2)$.

[7] X Ren, B Kuhlenkotte, H Muller. Simulation and verification of belt grinding with industrial robots. Intemational Joumal of Machine Tools \& Manufacture, 2006, 46(7-8): 708-716.

[8] X Ren, M Cabaravdic, X Zhang, et a1. A local process model for simulation of robotic belt grinding. Intemational Joumal of Machine Tools \& Manufacture, 2007, 47(6): 962-970.

[9] X Zhang, B Kuhlenkotter, K Kneupner. An efficient method for solving the Signorini problem in the simulation of free-form surfaces produced by belt grinding. International Journal of Machine Tools \& Manufacture, 2005, 45(6): 641-648.

[10] X Zhang, K Kneupner, B Kuhlenkotter. A new force distribution calculation model for high-quality production processes. Advanced Manufacturing Technology, 2005, 27(7): 726-732.

[11] A Khellouki, J Rech, H Zahouani. The effect of abrasive grain's wear and contact conditions on surface texture in belt finishing. Wear, 2007, 263(1-6): 81-87.

[12] A Khellouki, J Rech, H Zahouani. Influence of the belt-finishing process on the surface texture obtained by hard turning. Proceedings of the Institution of Mechanical Engineers Part B: Journal of Engineering Manufacture, 2007, 221(7): 1129-1137.

[13] S Mezghani, M E Mansori, H Zahouani. New criterion of grain size choice for optimal surface texture and tolerance in belt finishing production. Wear, 2008, 266(5).

[14] R Q WANG. The modeling and experimental research of belt grinding process in rail. Beijing: Beijing Jiaotong University, 2016.

[15] C L Wu, H Y Ding, Y Chen. Research on modeling method of material removal depth in $\mathrm{CNC}$ mechanical polishing for aluminum 
alloy wheel. China Mechanical Engineering, 2009, 20(21): 2558-2562.

[16] C L Wu, H Y Ding, Y Chen. Research on modeling method of relation between abrasive grain and material removal depth. China Mechanical Engineering, 2011, 22(3): 300-304.

[17] W Wang, F Liu, Z Liu, et al. Prediction of depth of cut for robotic belt grinding. International Journal of Advanced Manufacturing Technology, 2017, 91(1-4): 699-708.

[18] G Xia, Y Huang. Adaptive belt precision grinding for the weak rigidity deformation of blisk leading and trailing edge. Advances in Mechanical Engineering, 2017, 9(10): 1-12.

[19] Y Wang, Y Huang, Y Chen, et al. Model of an abrasive belt grinding surface removal contour and its application. International Journal of Advanced Manufacturing Technology, 2016, 82(9-12): 2113-2122.

[20] W X Wang, J Y Li, W G Fan, et al. Abrasion process modeling of abrasive belt grinding in rail maintenance. Journal of Southwest Jiaotong University, 2017, 52(1), 141-147.

[21] W X Wang, J Y Li, W G Fan, et al. Grinding power prediction model for abrasive belt rail grinding based on Hertzian contact. China Railway Science, 2017, 38(3): 25-30.

[22] W G Fan, Y M Liu, W X Wang, et al. Research on modeling method of material removal for rail grinding by abrasive belt based on elastic Hertzian contact. Journal of Mechanical Engineerin, 2018, 54(15): 191-198.

[23] W G Fan, J F Cheng, H B Lü, et al. Research on time-varying contact behavior and simulation for waved rail surface grinding by abrasive belt. Journal of Mechanical Engineering, 2018, 54(4): 87-92.

[24] W G Fan, J F Cheng, Y F Wu, et al. Research on Static Contact Behavior and Simulation for Rail Grinding by Abrasive Belt Based on Concave Type Contact Wheel. Journal of Mechanical Engineering, 2018, 54(14): 152-158.

[25] J J Roseman. The principle of Saint-Venant in linear and non-linear plane elasticity. Archive for Rational Mechanics and Analysis, 1967, 26(2).

[26] L P Valentin. Contact Mechanics and Friction Physical Principles and Applications. Tribology Letters 40.3 (2010).

[27] L D Landau, E M Lifschitz. Theory of Elasticity (Theoretical Physics, Vol. 7), $3^{\text {rd }}$ edition, 1999, Butterworth-Heineemann, Oxford, 8-9.

[28] H Wang. Simulation and analysis of active chip eliminate process using open-structured abrasive belt for rail grinding. Beijing: Beijing Jiaotong University, 2019.

\section{Biographical notes}

Chang Qian, born in 1997, is currently a master candidate at Key Laboratory of Vehicle Advanced Manufacturing, Measuring and Control Technology, Ministry of Education, Beijing Jiaotong University, China.

Tel:18810394615 ; E-mail: 19121248@bjtu.edu.cn

Zhi-Wei Wu, born in 1994, is currently a PhD candidate at Key Laboratory of Vehicle Advanced Manufacturing, Measuring and Control Technology, Ministry of Education, Beijing Jiaotong University, China.

E-mail: 1756832926@qq.com
Guang-You Hou, born in 1994, is currently an engineer at China Academy of Railway Sciences. He received his master degree on Mechanical Manufacturing and Automation in Beijing Jiaotong University, China, in 2020.

E-mail: 17121243@bjtu.edu.cn

Wen-gang Fan, born in 1985, is currently an associate professor at Beijing Jiaotong University. He received his $\mathrm{PhD}$ degree from Beijing Jiaotong University, China, in 2012. His main research direction is rail grinding technology and equipment, digital manufacturing technology and equipment.

E-mail: wgfan@bjtu.edu.cn 